\title{
Understanding Professionals' Perspectives on Access to Care for Patients with Carcinoma of the Urinary Bladder in Scotland: A Qualitative Study
}

\author{
Khan Redzwan Habib \\ World Health Organisation, Brisbane, Australia \\ Email address: \\ khan.redz@gmail.com,habibk@who.int
}

\section{To cite this article:}

Khan Redzwan Habib. Understanding Professionals' Perspectives on Access to Care for Patients with Carcinoma of the Urinary Bladder in Scotland: A Qualitative Study. American Journal of Health Research. Vol. 4, No. 4, 2016, pp. 75-81. doi: 10.11648/j.ajhr.20160404.11

Received: May 8, 2016; Accepted: May 24, 2016; Published: June 4, 2016

\begin{abstract}
Urinary bladder carcinoma is the most frequently occurring cancer of the urinary tract. Like any other types of cancer the diagnosis, treatment and overall management of urinary bladder cancer is not only expensive but also time consuming. During all these procedures, patients face several barriers while accessing care. The physicians and healthcare administrative or policy makers have the opportunity to closely observe patients' situation or their view towards existing policy and support system. Objective of this study is to explore professionals' perspective on access to care for patients with urinary bladder carcinoma in Scotland in order to identify recent progresses and difficulties faced by patients at different stages of management of the disease. Semi structured interview approach was chosen for its suitability for exploring attitudes, values, beliefs and motives. A total of twelve participants were interviewed. All the twelve participants were chosen through purposive sampling. A phenomenological approach was used to explore perspectives of these twelve professionals having experience of treating patients with urinary bladder carcinoma. All the participants despite of the differences in their views have identified backlog and/or long standing waiting list, lack of patient centered care and shortage of infrastructure, as major barriers in access to care for urinary bladder carcinoma patients. The major gaps identified were inefficiencies of the system and lack of focus on bladder cancer. According to the participants' financial deficiency, systemic complication, age group and socioeconomic status of patients are the biggest challenges to overcome these barriers. Professionals gave their opinion for increasing the capacity of the service by establishing bladder cancer care centers and also employing skilled workforce in accordance to the patient number in these centers.
\end{abstract}

Keywords: Barriers to Care, Gaps in Service Delivery, Cancer Survival, Urinary Bladder Carcinoma

\section{Introduction}

Worldwide each year an estimated 382,660 new cases of bladder cancer are diagnosed. In terms of most frequently occurring cancers urinary bladder carcinoma is at number six. It is the most frequently occurring cancer of urinary tract accounting for 1 in every 30 cancer in the United Kingdom [1]. In Scotland urinary bladder carcinoma is the fifth most common cause of cancer in men and fifteenth most common among women. In the year 2010 in Scotland the number of new case diagnosed with urinary bladder cancer was 778 (male 542, female 236) and number of deaths recorded in 2011 were 472 (male 294, female 178). In Scotland between the year 2003 to $2007,55.1 \%$ of the male patients and $37.7 \%$ of the females diagnosed with bladder cancer were found to have survived for 5 years or over [2].

All the patients with bladder carcinoma despite of their cancer grading and treatment need to be on constant physicians' follow up as recurrence and progression is common in this type of cancer [3]. So even after complete recovery physicians regularly do follow up treatment in every 3 to 6 months to observe if the cancer growing back or is there any sign of new cancer progression [4]. Therefore, due to the increase in the year a patient has survived, it has an effect on treatment expenditure and the necessity for accessing to care has increased. This increased need for accessing to care also increases overall workload of the existing support structure, which interrupts access and flow 
of care by giving rise to various gaps and barriers in access to care.

Literature reviews reviled inadequacy of staffs and infrastructure [5-6], prolonged waiting time [7-9] and inequity in cancer care [10-11] as the major barriers in access to care for urinary bladder carcinoma patients. The gaps identified in the literature in service provision were ineffective support system [12-13] and financial inconvenience [14-16]. To find a suitable intervention it is important to understand the link between bladder cancer and the mentioned factors influencing cancer care. The clinical staffs and the healthcare administrators or policy makers remain in close contact with the patients and therefore have a practical experience about mentioned gaps and barriers and other factors influencing urinary bladder cancer care. While there have been studies about breast, lung and other urological cancer, professionals' perspectives about bladder cancer have not been investigated. The aims of this study were to investigate the professionals' perspectives about gaps and barriers in access to care and to inform policies for service improvements in urinary bladder cancer care in Scotland.

\section{Methods}

A phenomenological approach was chosen for this particular study as this method aims to understand the thoughts and experiences of a particular group of people involved in a particular action [17]. Rather than conducting research based on a preconceived frame or a prior hypothesis this study moved forward by collecting and generating data to build a new hypothesis. Semi structured interview approach was chosen for its suitability for exploring attitudes, values, beliefs and motives [18]. A minimum number of 10-15 participants were sought to recruit through purposive sampling. The involvement of expertise's in healthcare and related support systems is summarized by Dancey, et al. [19] and Riechelmann, et al. [20] as they focused on the involvement of social workers, policy makers side by side with GPs, specialist physicians and trained nurses on specialized fields. Therefore, over a time of one month GPS, specialist nurses, consultant urologists and policy makers were recruited from different hospitals and healthcare organizations in Scotland.

All potential interviewees were first contacted over emails carrying a copy of invitation letter, a participant information sheet and a consent form. The consent form and information sheet carried clear information about the study background and what the participants have to do if they decide to participate in the study. All the interviews were one to one interviews. One to one interviews were chosen as they are more suitable to identify the "essence" of human experiences concerning such specific phenomena whilst remaining true to the description and perspectives of the participants in the study [21]. The topic guide was developed based on the findings emerged from relevant literature. The key areas of concentration in the interviews are summarized in Box A.
Box A: Key areas of concentration in the interviews:

I Factors influencing cancer care:

a) Low socio-economic status

b) Patient location/Remote areas

c) Ethnicity

II Demands of treatment:

a) Physical demands

b) Social and psychological demands

c) Financial and economic demands

IIIModels of care

IVPolicy context in urinary bladder carcinoma treatment

$\mathrm{V}$ Gaps in research

VIWhat is needed now

All interviews with participant's consent were audio taped for further analysis and were disposed at the end of the study. The transcripts were analyzed using NVivo-9. The analysis was performed according to Braun and Clarke's guideline of 'Six phase of coding' qualitative data [22]. Five major issues of concern according to Kendall, et al. [23] while accessing to care for 'cancer patients (eg. Urinary bladder cancer) were considered during data analysis: information, communication, equity, holistic approach and patient centered care.

The study received ethical approval from Ethics Review Group, Centre for Population Health Sciences, The University of Edinburgh.

\section{Results}

A total of twelve interviews were conducted among which two were phone interviews and the rest were conducted face to face. Among the twelve participants two were urologists, one was a registrar in a hospital urology department, four were General Physicians, four were specialist nurses (one flexible cystoscopy, one stoma and two urology nurse specialist) and the remaining participant was a healthcare policy maker.

An overview of participants' characteristics is given in table 1 . All but one participant had the experience of working in respective fields for over 10 years. Only one of the total participants was retired. On an average all the participants except for the GPs were identified to deal with around 10 patients per week. To maintain anonymity the participants were assigned with two identification letters for each: specialist physicians / consultants of urinary bladder carcinoma were denoted 'CU'; registrar in urology was denoted 'RU'; specialist nurses were denoted 'SN'; GPs were denoted 'GP' and the healthcare administrator was denoted 'HA'. Numbering of the individual participants was done chronologically depending on the interview date.

Five themes emerged from the data regarding access to care for patients with urinary bladder carcinoma in Scotland: barriers to care; gaps in service provision; reasons for persistency; service improvements and recent progress. Influencing factors regarding access to care for urinary bladder carcinoma patients in Scotland are discussed in below. 
Table 1. Over view of study participants.

\begin{tabular}{llll}
\hline $\begin{array}{l}\text { Participant } \\
\text { code }\end{array}$ & Type of expertise & $\begin{array}{l}\text { Year of } \\
\text { experience }\end{array}$ & $\begin{array}{l}\text { Patients seen } \\
\text { per week }\end{array}$ \\
\hline CU1 & Consultant urologist & 12 & $5-15$ \\
CU2 & Consultant urologist & 17 & $8-15$ \\
RU & Registrar urology & 6 & $10-15$ \\
GP1 & General physician & 11 & $1-3$ \\
GP2 & General physician & 13 & Around 3 \\
GP3 & General physician & 14 & $1 / 2$ \\
GP4 & General physician & 11 & $1 / 2$ \\
SN1 & Nurse specialist & 14 & Around 12 \\
SN2 & Nurse specialist & 11 & $8-12$ \\
SN3 & Nurse specialist & 13 & $5-12$ \\
SN4 & Nurse specialist & 13 & $5-10$ \\
HA & Health policy maker & 16 & - \\
\hline
\end{tabular}

\subsection{Barriers to Care}

All the professionals despite of the differences in their views have identified three major barriers in access to care for urinary bladder carcinoma patients. The key barriers are: backlog and/or long standing waiting list, lack of patient centered care and shortage of infrastructure.

The reasons behind backlog to persist according to the interviewees were different. The main areas of concern were: miscommunication between the office stuffs and patients, scarcity of skilled clinical and office stuffs and delayed referral. Physicians and nurses agreed that, administrative staffs need to be more mindful about the patient's situation and give attention to details. From the professionals' perspectives the reason behind miscommunication may be their lack of clinical knowledge, "If we depend on the admission office you can't get this sort of fine-tuned service...... They cannot differentiate between a muscle invasive or metastatic cancer and therefore the process may be delayed for patients who needs immediate care" (CU1)

The clinical staffs and also the administrator pointed out the shortage of stuffs in NHS as a historical fact. The shortage of stuffs plays a vital role in the development and persistence of long waiting lists, "the hospitals are under resource in terms of both operating time and surgeons to get them through quickly. The departments are under resourced in terms of clinical staffs and clinical resource" (RU). Also some blamed lack of appropriate training to existing staffs. According to some professionals' providing specialist training to existing staffs may help to tackle this situation for the time being till new staffs are employed.

"(while talking about a nurse specialist who was taken off from her duty station for unknown reason)..... I don't know what the reason was but it is not difficult to train another nurse to take her place" (SN3)

Complexity of the access to care often turns out to be troublesome and time consuming for the patients seeking to access for care. Therefore the system itself often becomes the reason for agitation to the patients. "Our system only allows booking an appointment 4-6 weeks ahead of time. I think it would be good if the patients could come for a cystoscopy and on the same day could book an appointment for their next follow up" (SN1).
Bladder carcinoma patients physically as well as mentally are vulnerable like any patients of chronic diseases. The clinical and the administrative staffs, all expressed their opinion about dealing with such vulnerable patients with more delicacy. Any slightly insensitive attitude from the clinical staffs may worsen already degrading mental strength of these patients, "(talking about a patient who was unhappy about the way she was confirmed about her diagnosis) according to her their (hospital staffs) behavior was uncongenial and they seemed like they are in a hurry...... while informing a patient with such diagnosis people in charge should be a little more sympathetic" (GP2). Clinical staffs also pointed out to an interesting fact about patients' psychology about presenting their complaints, which sometimes delay the process of referral.

"...... they often think they will tell the consultants their complaints at once...... mostly they want to be referred immediately I understand their concerns but there is a protocol" (GP3)

All the clinicians emphasized that to run the delivery of care smoothly and flawlessly there is no other way than increasing the capacity of infrastructure. In some cities there is only one hospital with capacity to offer specialized procedures like flexible cystoscopy.

".......why you have to come thirty miles for a two minutes procedure...... the whole bladder cancer care service needs to be decentralized...... to provide better service which we always say is, care locally for the patients when possible" (CU2)

Professionals also identified some problems faced by the ethnic minority groups while accessing the care system for bladder cancer. They identified it not only as a problem of the system itself but also sometimes lack of awareness or cooperation for ethnic minority population, "the difficulty for them is because of their cultural background and language barrier...... specially the women" (CU2)"

\subsection{Gaps in Service Provision}

The major gaps in access to care for bladder carcinoma patients identified were: inefficiencies of the system and lack of focus on bladder cancer. Recent years witnessed the development of several support groups for bladder carcinoma patients. The clinicians have expressed that despite their efforts a large number of patients are not aware of these support groups.

"We diagnose the disease but sometimes incomplete and inadequate information, difficulties in addressing patients' perception of fear remains undiagnosed that is where they can use moral or psychological supports"......in recent years several patient support group for bladder carcinoma patients have been established..... but not a lot of people know about it" (CU2).

Clinicians also have talked about some of the confusing situations they face themselves when it comes to the point of supporting suspected but unconfirmed bladder cancer patients. The whole procedure from cystoscopy to biopsy takes 4-6 weeks and more two weeks for the biopsy result to 
arrive. Clinicians mentioned this time frame very crucial for the patients and expressed that they themselves are confused sometimes about how to support these patients as they don't know the result is going to be positive or not.

"The patients are very anxious for potential diagnosis and we understand their state of mind but how can you support someone when you don't know that they have cancer or not" (SN2).

Participants expressed their despondency with the fact that urinary bladder carcinoma being one of the most common types of all carcinomas is not highlighted the same way. Even the relation of common habits like smoking with bladder carcinoma does not often get the same media coverage.

"A lot of people don't know about bladder cancer. There is a lot of campaign about breast, lung or prostate cancer or the connection of smoking to lung cancer. But most of the people don't know that there is a relation between bladder cancer and smoking" (SN3).

\subsection{Reasons for Gaps and Barriers to Persist}

The interviewees identified shortage of finance as the biggest obstacle to overcome the challenges faced in improving the access to care. Interviewees believe that if one specific health problem is given more focus there might be chances for other problems to be over looked, "The difficult thing is with the current finance it is difficult to focus on one disease and as a result another will be over looked" (HA)

Overcoming resistances within NHS system sometimes is a big challenge itself. As any new decision needs to go through bureaucratic pathways before it is finally implemented it gives rise to inertness and infirmity.

"Any improvement of the service always meets with resistance from the stuffs, rigidity of NHS system and convincing the stack holders that what improvement a real redesign may make...... sometimes the problem is a redesign is made only redesigns sake (CU2)"

Age of the affected patients plays a vital role in different steps of disease management especially in development of charitable foundation, attracting media highlight and over all to assure flawless access to care. The professionals expressed that urinary bladder carcinoma usually affects the older patients and this is unfortunately one of the major reasons this cancer not receiving media coverage and public attention like other types of common cancers, comparing to other cancers it (bladder cancer) is more of a common disease to elderly and social deprived group of people. Whereas majority of prostate or breast cancer patients are comparatively younger and more privileged who have the courage to start a movement so these are more talked about, more advertized......" (RU)

\subsection{Service Improvements}

Participants had different thoughts about how access to care can be improved but they all agreed on one point that it requires team work, a specialized Centre with proper skilled staffs, establishing more one stop clinics for bladder cancer care and dedication from the clinicians' side, "More dedicated clinicians, nurses, office staffs and operation theaters are needed..... proper co-ordination between GPs, urology, oncology, radiology is needed so that the patients do not need to come every other day" (GP4).

Interviewees also emphasized on establishing a urinary bladder cancer center solely dedicated to care for the bladder cancer patients.

"...... what I would like to see in a utopian world to provide even better care to the patients 'a bladder cancer center', where we have dedicated consultants, supported by specialist nurses trained on bladder cancer management, administration office who will understand the importance of disease category, a patient support group and a secretarial assistance who are specially trained to keep communication between the clinical team and bladder carcinoma patients. In short we need a dedicated bladder cancer aspect of the service within the service" (CU1)

Establishing more one stop clinics would also be very useful interviewees said. A one stop clinic acts as a primary care hub where patients can get all types of emergency supports, information and relevant tests done at once on the same day.

"I have seen in my own experience that one stop clinics are better as they can come on one day

and seen and reviewed by specialized nurses, go to the radiology have their scan and then go to see one of the doctors for cystoscopy and reviewed which makes things a lot more easier for the patients" (RU)

Some of the interviewees expressed that the first priority should be to identify the main underlying reasons for the gaps and barriers to persist rather than blaming shortage of finance or stuffs. They argued that not always the underlying problem can be solved by recruiting more stuffs or ensuring more funding.

"There is a scope for redesigning the service..... the first and foremost is to find where the significant bottle necks are...... is it in the initial diagnostic pathway or is it with the patients' anxiety or lack of proper information leading to their fear or slight dissatisfaction with the treatment they are getting" (CU2)

\subsection{Recent Progress}

All the participants in this study agreed that in last few years there have been noticeable improvements in the access and delivery of care for the urinary bladder carcinoma patients. Professionals' expressed that improvement in referral system, availability of test results and also appointments for follow up has been very satisfactory.

"Patients in last 6-8 years have been a lot better cared for, getting much more information than they used to. May be the new patients won't see the difference but if you talk to any of the old patients they will be able to tell you the big difference. We call the whole procedure CIS (Communication, Information and Support)" (CU1)

There have been several publications, internet websites and also back up booklets for the patients now to gather 
information about their disease and to know important facts like side effects while going through chemo or radiotherapy. The clinicians also make effort to make the patients better informed about this available information.

"..... I also provide them with back up booklets (Understanding superficial bladder cancer: Macmillan back up booklet)...... What I have also started doing recently is to give a leaflet of Maggie's Center with the Macmillan backup book let. This is a nice place for relaxation and time out for the cancer patients" (SN4).

Another achievement was establishment of several charitable foundations. These foundations provide the patients with not only information about the disease but at times with valuable moral and psychological support. Also the government is organizing nationwide programs to keep people better inform and for earlier diagnosis.

"There is a Scottish government program coming soon called 'Blood in Pea', the plan is to detect urinary bladder cancer early...... as we do not know what proportion of men or women in the community report it to their GPs after they notice blood in their urine, I think this is a good step" (CU2).

\section{Discussion}

Participants of the study have identified several potential barriers in access to care for patients with urinary bladder carcinoma. The last policy reform has obliterated many historical problems the patients used to face in this field. But as found in the literature review long standing waiting list [79] still seems to be the major obstacle for the patients with urinary bladder carcinoma seeking for accessing to care.

A number of interviewees blamed miscommunication between hospital staffs and patients and lack of clinical knowledge as the hospital staffs for this. Due to their lack of clinical knowledge as the hospital staffs tend to deal patients of all stages of cancer in the same manner. A strict national stipulated guideline is present for the patients to be seen by a consultant physician within a certain time frame. But sometimes it happens that the patients miss the appointment date or the patient who needs to be seen by the consultant urgently are put behind the patients for whom seeing the consultant is comparatively less urgent. Professionals also they gave their opinion for the patients to express all their complaints to GPs starting from the very first visit rather than waiting to be referred to the specialists and tell it to them.

According to the literature review [5-6] shortage of clinical staffs was another reason for the barrier with long standing waiting list to persist. The professionals' perspective about this issue was plain and simple. The number of bladder carcinoma patients are increasing every year, so is increasing patient survival time, but the number of clinical staffs are inadequate to handle this increased patient demand. Shortage of infrastructure was identified as another major obstacle in the literature [24] in way to ensure smooth and continual access to care for the patients with urinary bladder carcinoma. The experts supported this theory as they expressed that believe increasing the capacity of the service would help them to perform surgical procedure within an ideal time frame and would eventually be beneficial for the bladder carcinoma patients.

It was also not a beneficial decision for the patient to shut down specialized services in some hospitals. As a result one single hospital and even worse than that, in some cases one trained person has to deal with almost twice the patient load which experts believe may lower the quality of service and would make the waiting lists longer. Not only that, it will eventually increase patients' travelling time to get specialized care. Though it has not been sufficiently highlighted like other common factors as a barrier to care in the literature; inefficiencies of the system like poorly organized care pathways or episodes of care resulting in patient distress can be considered as another major barrier in access to care for bladder carcinoma patients. It would be easier on the patients' part if they can do all the necessary tests in one day unless there is any clinical barrier that they have to wait. But current system in the hospitals does not let the patients perform all activities regarding their treatment management on the same day. Though these types of complain are very much unlikely but sometimes patients become victims of insensitive behavior of the hospital staffs. This may dishearten the patients and may further damage their already degraded mental strength.

From the literature review [25] it was identified that ethnicity plays an important role in bladder cancer management. Professionals' also spoke of this during the interview as they have agreed that for the patients from ethnic minority groups language barrier and cultural beliefs also play a vital role for getting diagnosed at a later stage. Their lack of awareness about bladder cancer and failure to understand seriousness of the disease and symptoms sometimes negatively contribute to disease management.

Many of the studies [13, 26] talked about lack of availability of support providers. But in this study it was found that in the present situation rather than availability of support providers lack of awareness among the patients about these supports services are bigger concern and a gap in service provision that needs to be bridged in order to ensure flawless access to care. The study participants mentioned the eight weeks' time frame from cystoscopy to biopsy very crucial for the bladder carcinoma patients. They have also expressed their confusion how someone suspected for cancer but not being diagnosed can be supported. Finding a way to provide moral support to these potential patients is of great importance. It was found in the literature 12 and also was found from the professionals' perspectives that even after diagnosis, to assure the patients and guiding them to choose right path of treatment is very crucial. Support is also needed when these patients after surgical procedures like TURBT (Trans-urethral resection of bladder tumor) tries to cope up with stoma and their changed life style as operations like this may alter normal physiological systems.

According to the professionals' lack of focus from the media towards bladder cancer plays an important role for the general population being relatively unaware about this 
disease. Any type of carcinoma is life threatening, therefore same focus from the end of social media is expected to raise awareness about these diseases. But bladder cancer according to the study participants is not receiving the same focus in the media like other common cancers.

Though everyone agreed that recent NHS policy about urinary bladder carcinoma has been very useful for the patients but experts identified several potential challenges to overcome to make access to care more patient friendly. But for NHS, funding all the sectors or diseases in the same manner is not easy. If focus is put on one specific disease there is chance of other diseases getting overlooked. Professionals also argued that not only adequate finance is the reason for gaps and barriers to persist. According to them unreasonable behavior of the staffs and rigidity of the major stakeholders (eg. legislative and executive bodies within the government, employers' organizations, private for profit or community based organizations etc.) also contribute to the persistence of these barriers in the system. Therefore, it is important to set priority and maintain accordance.

Studies [10-11, 27] have identified low socio-economic status as a reason for inequity in access to cancer care but the study participants talked about inequity issue from a relatively different angle. First of all patients of urinary bladder carcinoma are comparatively older than other carcinoma patients. Also this type of carcinoma according to the experts is more common in socially deprived population. Therefore, there is both lack of courage and also lack of awareness among this type of carcinoma patients to attract media coverage or to start a social movement against their disease.

There seem to be a significant demand for employing more skilled clinicians and to increase capacity of service. There is apparently a shortage of skilled clinical staffs from the root level up to specialist physicians for bladder cancer. But just employing more people is not the end of the problem. As found in the studies [19, 26] and also supported by the professionals that there need to be a good co-ordination among all the specific departments like urology, chemotherapy and radiology. Co-ordination of services and teamwork is important to make access to care patient friendly and efficient.

Professionals are found to be a bit unsure of the current separate guidelines for frank and microscopic hematuria. Some of the professionals think there the current guideline needs to be restructured and few were found uninformed and unaware of two separate guidelines. There seemed to be a gap in the communication about guidelines among the professionals. Introducing two separate guidelines and two different patient slots improved the current access to care situation but at the same time it increased the workload. Therefore according to professionals' perspectives the way out of this problem will be to establish a shared care center, more primary care hubs and a skilled workforce to understand patient need.

Several studies focusing on patients' or clinicians' perspectives about breast, skin or prostate carcinoma have been identified. But no such study regarding urinary bladder carcinoma was found. It is therefore worthwhile to conduct further research to identify is this type of carcinoma really under publicized and if so what are the reasons. Also research is needed to determine the cost benefit ratio if more funding is provided for this disease.

\section{Conclusion}

Long standing waiting list, lack of patient centered care and the study participants as the major obstacles in access to care for bladder carcinoma patients identified lack of highlight on bladder cancer. As discussed in this study according to the professionals the reasons for these gaps and barriers to persist were many. Shortage of staffs and infrastructure, inefficiencies of the system and financial or systemic barriers were found to play an important role for these barriers and gaps to persist. Professionals gave their opinion for restructuring the existing protocol and increasing the capacity of the service by establishing a cancer care center and more one-stop primary care hubs. Also employing skilled workforce in these centers would eventually help to overcome patients' complains. Though they have agreed that there is limitation of finance but it has been anticipated that implementing mentioned policies in this study will make access to care more patient friendly and efficient. Professionals gave their opinion for increasing the capacity of the service by establishing bladder cancer care centers and also employing skilled workforce in accordance to the patient number in these centers.

Based on the findings of the study, it is proposed that further research is needed regarding perspectives of the patients of Urinary bladder carcinoma to have in depth understanding of the survivors' difficulties faced while accessing to care and finding ways to ensure better support for these patients. Practical implications of patients with urinary bladder carcinoma are listed.

\section{References}

[1] Cancer Research UK. Bladder cancer incidence statistics. 2012. http://www.ancerresearchuk.org/cancerinfo/cancerstats/types/bladder/incidence/uk-bladder-cancerincidence-statistics (Accessed 6 September, 2013)

[2] The Scottish Government. Better cancer care, an action plan. Edinburgh: The Scottish Government. Report number: ISBN: 978-0-7559-5857-3, 2008.

[3] Pasbos CL, Botteman MF, Laskin BL and Redaelli A. Bladder Cancer Epidemiology, Diagnosis, and Management. Cancer practice, 2002; 10(6): 311-321.

[4] American Cancer Society. Bladder cancer overview: US. 2012. $\mathrm{http}$ ://www.cancer.org/cancer/bladdercancer/overviewguide/b1 adder-cancer-overview-after-follow-u-p. (Accessed 17 December 2012).

[5] Obrey A, and Vasso V. The lived experience of overseas black and minority ethnic nurses in the NHS in the south of England. Diversity in Health and social care, 2004; 1(1): 13-20. 
[6] Rachet B, Woods LW, Mitry E, Riga M, Cooper M, Quinn MJ, Steward J, Brenner H, Este've J, Sullivan R and Coleman MP. Cancer survival in England and Wales at the end of the 20th century. British Journal of Cancer, 2008; 99: S2-S10.

[7] Richmond C. NHS waiting lists have been a boon for private medicine in the UK. Canadian Medical Association Journal, 1996; 154(3): 378-381.

[8] The Scottish Government. Long Term Conditions Collaborative Improving Care Pathways. 2010. http://www.gov.scot/resource/doc/309257/0097421.pdf (Accessed 17 December 2012)

[9] Wait list management project group. Waiting too long: reducing and better managing wait times. 2006. https://www.doctorsofbc.ca/sites/default/files/waiting_too_lon g.pdf (Accessed 8 September, 2013).

[10] Underwood W, Dunn RL, Williams C, and Lee CT. Gender and geographic influence on the racial disparity in bladder cancer mortality in the US. Journal of American College of Surgeons, 2006; 202: 284-290.

[11] Shack LG, Rachet B, Brewster DH and Coleman MP. Socioeconomic inequalities in cancer survival in Scotland 1986-2000. British Journal of Cancer, 2007; 97: 999-1004.

[12] Corbin J and Strauss A. Managing chronic illness at home: three lines of work. Qualitative Sociology, 1985; 8: 224-247.

[13] Curtis JR, Wenrich MD and Carline JD. Patients' perspectives on physician skill in end-of-life care: differences between patients with COPD, cancer, and AIDS. Chest, 2002; 122 (1): 356-362.

[14] Langa KM, Fendrick AM and Chernew ME. Out of pocket health care expenditures among older Americans with cancer. Value Health, 2004; 7: 186-194.

[15] Sangar VK, Ragavan N, Matanhelia SS. The economic consequences of prostate and bladder cancer in the UK. British Journal of Urology, 2005; 95: 59-63.

[16] Yabroff KR, Davis WW and Lamont EB. Patient timecosts associated with cancer care. Journal of National Cancer Institute, 2007; 99: 14-23.
[17] McLeod SA. Qualitative Quantitative Simply Psychology. 2008. http://www.simplypsychology.org/qualitativequantitative.html (Accessed: 9 September, 2013)

[18] Barriball KL and White A. Collecting data using a semistructured interview. Journal of advanced nursing, 1994; 19 (2): 328-335.

[19] Dancey A, Rayatt S, Courthold J and Roberts J. Views of UK melanoma patients on routine follow up care. British Journal of Plastic Surgery, 2003; 58: 245-250.

[20] Riechelmann RP, Tannock IF and Wang L. Potential drug interactions and duplicate prescriptions among cancer patients. Journal of National Cancer Institute, 2007; 99: 592-600.

[21] Creswell JW. Research design: Qualitative, quantitative, and mixed methods approaches (2nd ed). New York: SAGE Publications; 2008.

[22] Braun V and Clarke V. Using thematic analysis in psychology. Qualitative Research in Psychology, 2006; 3 (2): 77-101.

[23] Kendall M, Boyd K, Campbell C, Cormie P, Fife S, Thomas $\mathrm{K}$, Weller D and Murray SA. How do people with cancer wish to be cared for in primary care? Serial discussion groups of patients and carers. Family Practice, 2006; 23: 644-650.

[24] The Royal College of Radiologists. A national audit of waiting times for radiotherapy. 1998. https://www.rcr.ac.uk/publications.aspx?PageID=149\&Publica tionID $=50$

[25] Jiwa, M., Saunders, C. M., Thompson, S. C., Rosenwax, L. K., Sargant, S., Khong, E. L., Halkett, G. K. B., Sutherland, G., Ee, H. C., Packer, T. L., Merriman, G. and Arnet, H. R., 2008. Timely cancer diagnosis and management as a chronic condition: opportunities for primary care. Medical Journal of Australia, 189(2), 78-82.

[26] Kevin, C., Oeffinger, M. and McCabe, M. S., 2006. Models for Delivering Survivorship Care. Journal of Clinical Oncology, 24(32), 5117-5124.

[27] Audit Scotland, 2012. Health inequalities in Scotland Prepared for the Auditor General for Scotland and the Accounts Commission Scotland: Auditor General for Scotland. 